\title{
Measurements of the thermal diffusivity and conductivity of magnetic microwires using lock-in thermography
}

\author{
by R. Fuente*, A. Salazar*, A. Mendioroz*, A. Zhukov** and V. Zhukova**
}

\begin{abstract}
*Departamento de Física Aplicada I, Escuela Técnica Superior de Ingeniería, UPV/EHU, Alameda Urquijo s/n, 48013 Bilbao, Spain, agustin.salazar@ehu.es

**Departamento de Física de Materiales, Facultad de Químicas, UPV/EHU, Paseo Manuel de Lardizabal 3, 20018 San Sebastián, Spain, valentina.zhukova@ehu.es
\end{abstract}

Recent tendency in miniaturization of magnetic sensor devices requires development of novel advanced magnetic materials with improved magnetic properties. Among them a family of thin wire with reduced geometrical dimensions (metallic nucleus of order of 1-30 $\mu \mathrm{m}$ in diameter) gained special importance within last few years [1]. Particularly, it is reported on development of a number of magnetic sensors based on giant magneto-impedance (GMI) effect and stress-impedance (SI) effect with the C-MOS IC circuitry and advantageous features comparing with conventional magnetic sensors[1]. Main possible applications are related with the detection of the magnetic fields, small weights and vibrations, and such branches of the industry as the car industry and medicine are main consumers of these sensors. For many applications thermal properties of such microwires are quite important. Therefore we performed studies of the thermal diffusivity and conductivity of magnetic microwires.

Lock-in thermography is specially suited to study the lateral heat propagation in a wide variety of materials. In a typical setup, a modulated laser beam is focused onto the sample surface while an IR video camera records the surface temperature. Under ideal conditions (absence of heat losses and absence of diffraction effects), for a given modulation frequency $(f)$, both the natural logarithm of the amplitude of the temperature, $L n(T)$, and its phase $(\Psi)$ depend linearly on the distance to the heating spot, with the same slope given by $m=-\sqrt{\pi f / D}$, from which the thermal diffusivity $(D)$ can be obtained (the so-called slope method). In the case of thin films and thin filaments the slope method produces an overestimation of the thermal diffusivity due to the effect of heat losses. In order to eliminate the disturbing effect of conduction and convection to the surrounding air, the sample is introduced in a vacuum chamber. The remaining heat losses by radiation produce a change in the slope of both $L n(T)$ and $\Psi$, in such a way that the product of both slopes satisfies $m_{\psi} \times m_{\operatorname{Ln}(T)}=-\pi f / D$, which is in fact a generalization of the slope method $[2,3]$. This procedure has been applied successfully to measure the thermal diffusivity of a wide variety of filaments, from good thermal conductors to thermal isolators and with diameters as thin as a few microns [3]. Moreover, in the case of coated filaments the slopes method gives an effective value of the thermal diffusivity of the two-layer system $\left(D_{\text {II }}\right)$ which follows the in-parallel thermal resistor model

$$
D_{\|}=\frac{K_{\|}}{C_{\text {eff }}}=\frac{K_{1} v_{1}+K_{2} v_{2}}{\frac{K_{1}}{D_{1}} v_{1}+\frac{K_{2}}{D_{2}} v_{2}}
$$

where $C$ is the heat capacity, $K$ is the thermal conductivity, $v$ is the volume fraction and subscripts 1 and 2 stand for the two layers of the filament. If the thermal properties of one of the layers are known, the thermal diffusivity and conductivity of the other layer can be retrieved.

In this work we apply this generalized slope method to characterize the thermal transport properties of metallic ferromagnetic microwires. These microwires, of the family XBSi where X: $\mathrm{Fe}, \mathrm{Co}, \mathrm{Ni}$, consist of a metallic core surrounded by a Pyrex coating, resulting in a full diameter of about 25 microns. The Pyrex coating may be removed by means of a concentrated HF solution. Besides, the original state of the cores of these microwires is amorphous, but once they reach a crystallization temperature, they transform into crystalline microwires, varying so their thermal properties.

We have used a suitable [3] lock-in thermography setup, provided with a vacuum chamber, to measure the temperature profile of the microwires heated by a modulated and focused laser beam. A complementary system consisting of a power supplier with two electrodes connected to the extremes of the microwires has been attached in order to crystallize them inside the very high vacuum chamber, by means of a current of a few $\mathrm{mA}$.

A sample of $\mathrm{C}_{77.5} \mathrm{~B}_{15} \mathrm{Si}_{7.5}$ microwire of core diameter of $17.8 \mu \mathrm{m}$ with a Pyrex $\left(K=1.12 \mathrm{~W} / \mathrm{mK}, D=0.65 \mathrm{~mm}^{2} / \mathrm{s}\right)$ coating making a full diameter of $22.8 \mu \mathrm{m}$ has been measured. First, thermal diffusivity of the amorphous coated microwire has been determined, Fig. 1, obtaining an effective value of $D_{\|}=1.53 \mathrm{~mm}^{2} / \mathrm{s}$. Then, the Pyrex coating has been removed and the bare metallic microwire has been measured, resulting in a thermal diffusivity value of $1.90 \mathrm{~mm} / \mathrm{s}$. Consequently, Eq. (1) lets us retrieve the thermal conductivity for the amorphous magnetic microwire, $\mathrm{K}=5.0 \mathrm{~W} / \mathrm{mK}$. As a second step, the crystallization of the microwire has been performed. Keeping the microwire in vacuum, two electrodes 
have been connected to the extremes of the microwire and a current of $20 \mathrm{~mA}$ has been conduced through the microwire for one hour. Afterwards, measurements have been performed again, now for the crystalline state. First, for the coated microwire, it results an effective thermal diffusivity value of $2.63 \mathrm{~mm}^{2} / \mathrm{s}$. Second, for the bare metallic microwire, a diffusivity of $3.35 \mathrm{~mm}^{2} / \mathrm{s}$ has been obtained. Thus, by means of Eq. (1) we obtain the thermal conductivity of the crystalline magnetic microwire, $K=10.1 \mathrm{~W} / \mathrm{mK}$.

As we may observe from results, both thermal diffusivity and thermal conductivity in the amorphous state are approximately doubled when turned into a crystalline state. This result indicates that the heat capacity remains constant, as expected since it is a property depending on the sample composition and not on its structure. Besides, results are in good qualitative agreement with values found in literature [4,5] for ribbon samples of this family of materials. Further measurements on different microwires of the same family are in progress.
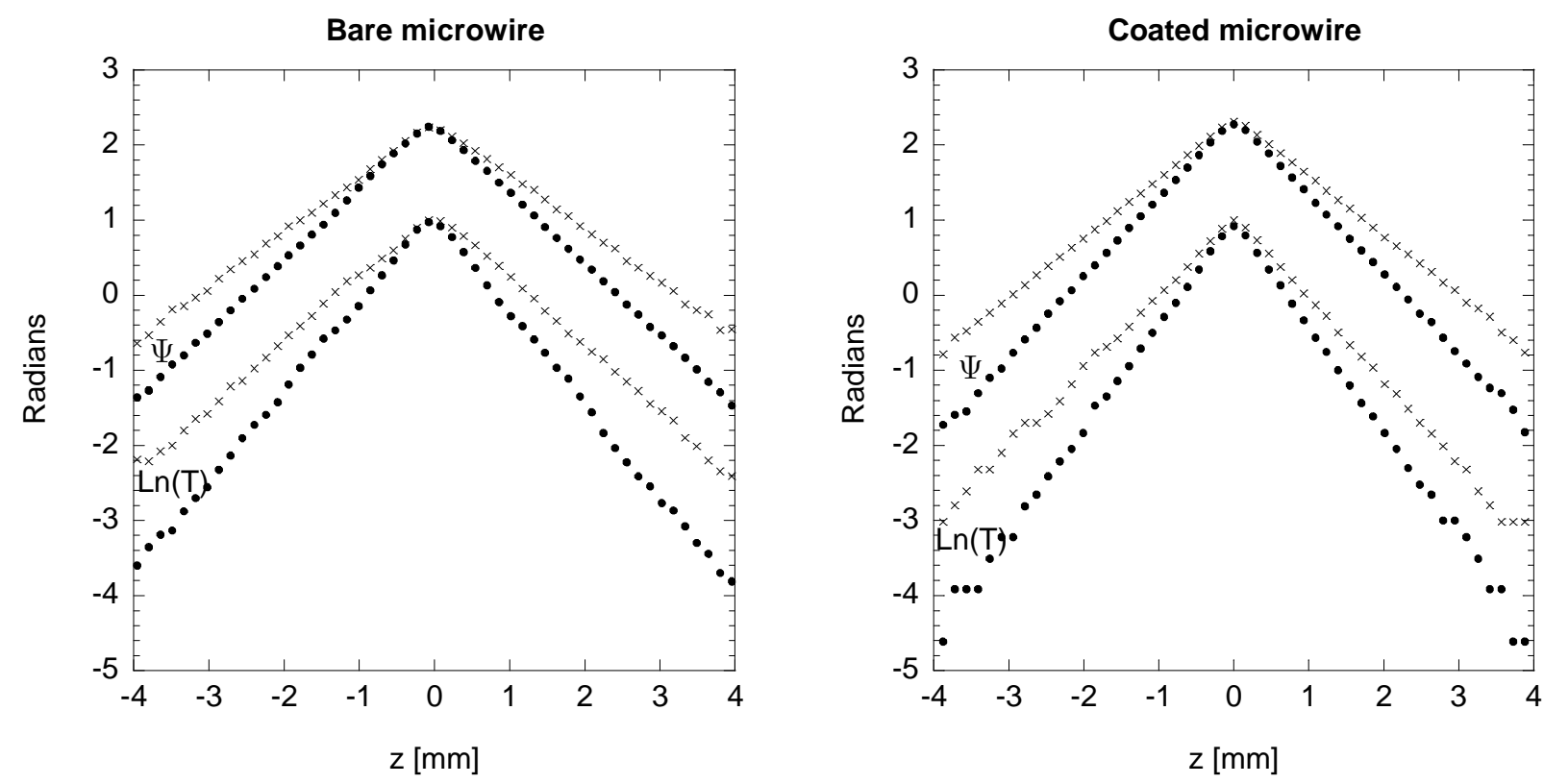

Fig. 1. Lateral dependence of $L n(T)$ and $\Psi$ at a frequency $f=0.71 \mathrm{~Hz}$ for a $\mathrm{Co}_{77.5} B_{15} \mathrm{Si}_{7.5}$ microwire of diameter $17.8 \mu \mathrm{m}$ (left) and a Pyrex coated version of diameter $22.8 \mu \mathrm{m}$ (right) in amorphous state (dots) and after crystallization (crosses). 18914).

This work has been supported by the Ministerio de Educación y Ciencia (MAT2008-01454 and MAT2010-

\section{REFERENCES}

[1] Zhukov A., Zhukova V., "Magnetic properties and applications of ferromagnetic microwires with amorphous and nanocrystalline structure". Nova Science Publishers Inc., New York, 2009.

[2] Pradère C., Goyhénèche J.M., Batsale J.C., Dilhaire R., Pailler R., "Thermal diffusivity measurements on a single fiber with microscale diameter at very high temperature". International Journal of Thermal Sciences, vol. 45, pp. 443-451, 2006.

[3] Salazar A., Mendioroz A., Fuente R., Celorrio R., "Accurate measurements of the thermal diffusivity of thin filaments by lock-in thermoagraphy". Journal of Applied Physics, vol. 107, pp. 043508-1-7, 2010.

[4] Choy C.L., Leung W.P., Ng Y.K., "Thermal conductivity of metallic glasses". Journal of Applied Physics, vol. 66, pp. 5335-5339, 1989.

[5] Choy C.L., Tong K.W., Wong H.K., Leung W.P., "Thermal conductivity of amorphous alloys above room temperature". Journal of Applied Physics, vol. 70, pp. 4919-4925, 1991. 